\title{
University of Toronto
}

From the SelectedWorks of Gustavo Saposnik

2012

\section{Bilateral Medial Medullary Infarction: A Systematic Review}

Gustavo Saposnik 


\title{
Bilateral Medial Medullary Infarction: A systematic review
}

Authors: Sujatha Parthasarathy MD* ${ }^{1}$, Jitphapa Pongmoragot MD* ${ }^{1}$, Daniel Selchen MD FRCPC

${ }^{1}$, Gustavo Saposnik MD MSc FAHA ${ }^{1-3}$

1 Stroke Research Unit, Division of Neurology, Department of Medicine, St. Michael's Hospital, University of Toronto, Canada

${ }^{2}$ Institute of Clinical Evaluative Sciences, Toronto, Ontario, Canada

${ }^{3}$ Departments of Medicine and Health Policy Management and Evaluation, University of Toronto, Ontario, Canada

Address for correspondence:

\author{
Dr. Gustavo Saposnik \\ 55 Queen St East, Suite 931 \\ St Michael's Hospital, University of Toronto, \\ Toronto M5C 1R6 Canada \\ Phone: (416)-864-5155 \\ e-mail: saposnikg@smh.ca
}

Conflict of interest: Nothing to disclose

* Both equally contributed to qualify as first authors. 
Running Title: Bilateral Medial Medullary Infarction: a systematic review

The present manuscript includes one figure and 3 tables. 


\begin{abstract}
Background: Bilateral infarction of the medial medulla (MMI) is rare. Limited information is available on clinical characteristics, etiology and prognosis. High resolution neuroimaging has a major role in elucidating the underlying stroke mechanism. The aim of this systematic review was to analyze the clinical presentation, stroke mechanism and outcome in patients with bilateral MMI.
\end{abstract}

Methods: We performed a systematic review of the literature from 1992 to 2011 that reported on clinical presentation, stroke mechanism and/or outcomes in patients with MRI proven bilateral MMI. Medline, EMBASE and Scholars Portal of Web Science were searched without language restriction. Two reviewers independently assessed studies to determine eligibility, validity and quality. The primary outcome was inpatient mortality and secondary outcomes were mortality at 12 months, an illustrative case is also described.

Results: We identified 138 articles from Medline, EMBASE, and Scholars' Portal including the above MESH terms. Twenty-nine articles met the inclusion criteria including 38 cases with bilateral MMI, who were included in our study. The mean age was 62.2 years, predominantly in male(74.2\%). The most common clinical presentations were motor weakness in $78.4 \%$, dysarthria 48.6\%, and hypoglossal palsy 40.5\%. Most common vascular pathology was vertebral artery atherosclerosis $38.5 \%$. The clinical outcome was poor (death rate $23.8 \%$, dependency $61.9 \%)$ 
Conclusion: Bilateral medial medullary infarction is a rare stroke syndrome. Clinical presentations were mostly rostral medullary lesions. Large artery atherosclerosis and branch disease were the most common stroke mechanisms. The clinical outcome was usually poor.

\section{Background}

Medial medullary infarction was first described in the19th century by Spiller. ${ }^{1}$ However, the initial description of medial medullary syndrome was attributed to Dejerine who proposed the clinical triad of ipsilateral hypoglossal paralysis, contraleteral hemiparesis sparing face and contralateral loss of deep sensation. ${ }^{2}$ In 1937 Davison first described the underlying vascular mechanism: the occlusion of the anterior spinal artery (ASA) and vertebral artery in MMI. ${ }^{4}$ Infarction of the medial medulla is uncommon and accounts for $0.5-1.5 \%$ of all strokes. ${ }^{3,5}$ Bilateral MMI is even rarer. Previously the diagnosis of bilateral MMI was from autopsies. More recently the advent of MRI revealed an increasing number of bilateral MMI cases. However, limited evidence is available on clinical presentation, stroke mechanism and outcome in patients with bilateral MMI. We have completed a systematic review of the literature analyzing clinical features, imaging, and prognosis in MRI proven patients with bilateral MMI.

\section{Methods:}

We reporting an illustrative case and performed a literature search in Medline, EMBASE, and Scholars' Portal Web of Science from 1992 to March 2011 on bilateral medullary infarctions. The search strategy combined the following Medical Subject Heading (MeSH) terms: 'brainstem infarctions', 'Medulla', and 'bilateral'. The results were then filtered to include only those citations with the keywords 'medial'. We also completed computer searches based on key words, and 
hand searched for references from previously retrieved articles. Articles were included if they met the flowing inclusion criteria: i) MRI proven bilateral medial medullary infarctions, ii) reporting on clinical presentation and vascular imaging, and iii) reporting on outcome measures (e.g. mortality or disability). The search was confined to human subjects and no language restriction was placed. We recorded demographic features, clinical presentation and neuroimaging (MRI and MRA brain).

\section{Study identification and validity assessment}

Two reviewers (SP, JP) assessed titles and abstracts to determine eligibility. Disagreements between raters were solved by a third reviewer (GS). Two reviewers (SP, JP) independently extracted data using standardized data collection forms. Information was collected on study design, participant characteristics, vascular imaging, and outcome measures. The primary outcome of interest was inpatient mortality. Independence at 2 months was a secondary outcome. Independence was defined by the modified Rankin scale (mRS) 0-2.

\section{Illustrative Case}

A 71 year old man with known atrial fibrillation not on anticoagulation, presented with acute onset of right sided weakness and garbled speech, NIHSS 6. CT and CT Angiogram revealed no brain abnormalities or vascular occlusions. IV tPA was administered within 3 hours of symptoms onset, without improvement. The following day he developed contralateral left hemiparesis, right horizontal gaze palsy and worsening of dysarthia, NIHSS 14. Although the tongue was central, he was unable to protrude it outside his mouth. There was no sensory impairment or ataxia. On the third day he became quadriplegic and had marked dysphagia 
requiring intubation. DWI MRI revealed acute infarcts in bilateral medial medulla at the rostral level, resembling a heart ("Heart sign") and an infarct in territory of the right posterior inferior cerebellar artery (PICA), sparing the lateral medulla (Figure 1A and 1B). Both CTA and MRA revealed diffuse intracranial atherosclerosis. The right PICA and anterior spinal arteries were not visualized. The left superior cerebellar artery was occluded on its second segment (Figure 1C). The echocardiogram revealed no obvious intracardiac thrombus. Considering the history of atrial fibrillation and multiple infarcts affecting different vascular territories, the most likely stroke mechanism was embolic either from the non-visualized spinal artery or cardiogenic (e.g. aortic arch). Unfortunately, the patient developed pneumonia and died.

\section{Results: Systematic review on Bilateral MMI}

We identified 138 articles from Medline, EMBASE, and Scholars' Portal Web of Science including the above MESH terms. Filtering with keywords yielded 61 citations for review.

Finally, 29 articles met the inclusion criteria, providing information on clinical and radiological aspects. Together these articles accounted for 38 patients with MRI proven bilateral MMI including our present case. We excluded Kim JS. et $\mathrm{al}^{6}$ from our analysis due to incomplete clinical information. We found no interventional studies or randomized controlled trial. There were seven articles published in languages other than English. Table 1 summarizes demographic information, clinical findings, imaging, and outcomes. The mean age was 62.2 years (range 4281). Men were most commonly affected (74.2\%). 


\section{Clinical findings:}

The most common clinical presentation was motor weakness $(78.4 \%)$, bilateral in $64.9 \%$ of patients. Other symptoms at onset included dysarthria and nystagmus in $48.6 \%$ (horizontal nystagmus $27 \%$, upbeat nystagmus $13.5 \%$, gaze evoke nystagmus $5.4 \%$ and rotatory nystagmus

2.7\%). Sensory disturbance was noted in $43.2 \%$. Hypoglossal palsy was present in $40.5 \%$ of patients, whereas dysphagia occurred in $16.2 \%$. One fourth of patients with bilateral MMI developed respiratory failure $(24.3 \%)$.

\section{Vascular imaging:}

Most common findings using MR angiography included: vertebral artery atherosclerosis $38.5 \%$, no abnormalities were found in $38.5 \%$, vertebral artery occlusion $15.4 \%$, basilar artery atherosclerosis $19.2 \%$. Dissection was reported in $7.7 \%$. Anterior spinal artery occlusion was noted in $3.8 \%$ ( from an autopsy case).

Outcome measures: Overall, the prognosis in reported patients with bilateral MMI was poor. In-hospital mortality was reported in $23.8 \%$, almost two third of survivors were dependent $(61.9 \%)$ at 2-3 months.

\section{Discussion}

Bilateral Medial Medullary Infarction (MMI) is a rare stroke subtype, usually presenting with sudden onset of quardriparesis/quardriplegia, loss of deep sensation, hypoglossal palsy, bulbar dysfunction, with or without respiratory failure. The initial diagnosis may be challenging due to the heterogeneity of the clinical presentation. The advent of MRI facilitates the diagnosis of 
medial medullary infarction. Most common mechanisms include large atherosclerotic vessel disease, small penetrating artery disease (ie. branch disease), and cardiogenic (e.g. cardiac source, aortic arch) seems the. Thirty-eight percent of patients reported in the literature had no abnormal vascular findings. Limited information is available in the literature regarding clinical presentation, stroke mechanism, and prognosis of bilateral MMI. To the best of our knowledge, our study is the largest systematic review including 38 MRI proven-cases with bilateral MMI (Table1). The most common clinical presentation was bilateral motor weakness $(64.9 \%)$ which corresponds to the site of the infarction (V shape). Interestingly, only $10 \%$ of patients with bilateral MMI presented with hemiparesis and $20 \%$ progressed from hemiparesis to quadriparesis within 72 hours. Nystagmus was also a common finding (48.6\%), upbeat nystagmus in $13.5 \%$ indicating the involvement of the medial longitudinal fasciculus. Respiratory failure was noted in $24.3 \%$, more prevalent than the reported $5 \%$ in unilateral MMI series. ${ }^{12}$ The most common MRI finding includes rostral medullary infarction (V-shape). Previous studies reported an initial negative DWI MRI in up to $20 \%$ of patients. ${ }^{5}$ In the comprehensive Posterior Circulation Stroke registry (New England Medical Centre Posterior Circulation study), cardioembolism was the most likely mechanism to cause distal posterior circulation territory infarcts ${ }^{11}$. In the largest reported case series of unilateral MMI $(n=86)^{6}$, large vessel disease was found in $62 \%$ (similar to our case) followed by small vessel disease in $28 \%$. Kim et al. ${ }^{6}$ also reported, Bilateral MMI (6 cases) vascular pathology was commonly found in atheromatous branch occlusion. Only $1 \%$ was found in cardiac embolism. In our study vascular pathology could be categorized in two major groups: large artery atherosclerosis involving the vertebral artery (38.5\%) and normal vascular imaging. The anterior spinal artery was non-visualized in $38.5 \%$. In the normal angiogram group the stroke mechanism was likely atheromatous branch occlusion or ASA occlusion which 
couldn't be demonstrated by MR angiogram. Bilateral MMI could be related either to extended thrombosis in the vertebrobasilar junction, or be due to the anatomical variability of perforator branches that supply the medulla or variation of anterior spinal artery originating from one of the VA may explain the occurrence of a simultaneous bilateral medial medullary infarction. Dissection of VA was uncommon in our study (7.7\%) compared with other reports of unilateral MMI. ${ }^{12}$ The clinical outcome was poor compared to unilateral MMI. ${ }^{3,6}$ In our study, one fifth of patients died and two third of survivors remained dependent.

The present systematic review has limitations. Symptoms were reported by different authors in a non-systematic fashion. Although we developed an algorithm to identify all cases with bilateral MMI, we cannot rule out an involuntary omission. Despite these limitations, our study summarizes the clinical presentation, imaging, and outcomes in patients presenting with bilateral MMI. A better understanding of this syndrome will assist clinicians to recognize bilateral MMI early, and to discuss therapeutic interventions and prognosis with patients and their families.

In conclusion, bilateral MMI is an uncommon stroke syndrome usually related to large artery or branch disease, and is commonly associated with poor outcome. 


\section{References}

1. Spiller WG. The symptom-complex of a lesion of the upper most portion of the anterior spinal and adjoining portion of the vertebral arteries. J Nerv Mednt Dis. 1908;35:775-77.

2. Dejerine J. Semiologie des affections du systeme nerveux. Paris: Masson: 1914: 226-230.

3. Toyoda K, Imamura T, Saku Y, Oita J, Ibayashi S, Minematsu K, et al. Medial medullary infarction: analyses of eleven patients. Neurology. 1996; 47:1141-7.

4. Davison C. Syndrome of the anterior spinal artery of the medulla oblongata. Arch Neurol Psychiat. 1937;37:91-107.

5. Shono Y, Koga M, Toyoda K, Matsuoka H, Yokota C, Uehara T, et al. Medial medullary infarction identified by diffusion-weighted magnetic resonance imaging. Cerebrovasc Dis. 2010;30:519-24.

6. Kim JS, Han YS. Medial medullary infarction: clinical, imaging, and outcome study in 86 consecutive patients. Stroke. 2009; 40:3221-5.

7. Toyoda K, Hasegawa Y, Yonehara T, Oita J, Yamaguchi T. Bilateral medial medullary infarction with oculomotor disorders. Stroke.1992; 23:1657-9.

8. Wada K, Gotoh T, Hashimoto Y, Kimura K, Uchino M. A case of bilateral infarction of medial pontomedullary junction. Rinsho Shinkeigaku. 1996; 36:1186-9.

9. Fukuda M, Aiba T, Takahashi S. Bilateral medial medullary infarction due to bilateral vertebral artery dissection. Clin Neurol Neurosurg. 2004; 106:132-5.

10. Bassetti C, Bogousslavsky J, Mattle H, Bernasconi A. Medial medullary stroke: report of seven patients and review of the literature. Neurology. 1997; 48:882-90.

11. Louis Caplan. Posterior Circulation Ischemia: Then, Now and Tomorrow: The Willis Lecture-2000. Stroke. 2000;31:2011-23.

12. Kameda W, Kawanami T, Kurita K, Daimon M, Kayama T, Hosoya T, et al. Lateral and medial medullary infarction: a comparative analysis of 214 patients. Stroke. 2004; 35:694-9.

13. Kleinert G, Fazekas F, Kleinert R, Schmidt R, Payer F, Offenbacher H, et al. Bilateral medial medullary infarction: magnetic resonance imaging and correlative histopathologic findings. Eur Neurol.1993; 33:74-6.

14. Ohashi T, Fujimoto M, Shimizu T, Atsumi T. A case of bilateral upper medial medullary infarction. Rinsho Shinkeigaku. 1995; 35:664-6. Japanese

15. Roh JK, Lee YS. Bilateral medial medullary infarction manifested as sensory ataxia: a case report and review of the literature. J Korean Med Sci. 1996; 11:193-6.

16. Del Brutto OH, Sánchez J, Mosquera A, Campos X. Bilateral medial bulbar infarct related to dolichoectasia of the vertebral artery. Rev Neurol. 1998;27:511-3. Spanish

17. Bailbé M, Rosier MP, Couderq C, Vandemarq P, Gil R, Neau JP. Bilateral medial medullary infarction. Rev Neurol (Paris). 2000; 156:384-7. French.

18. Katoh M, Kawamoto T. Bilateral medial medullary infarction. J Clin Neurosci. 2000;7:5435 .

19. Thijs RD, Wijman CA, van Dijk GW, van Gijn J.A case of bilateral medial medullary infarction demonstrated by magnetic resonance imaging with diffusion weighted imaging. $J$ Neurol. 2001; 248:339-40.

20. Kumral E, Afsar N, Kirbas D, Balkir K, Ozdemirkiran T. Spectrum of medial medullary infarction: clinical and magnetic resonance imaging findings. J Neurol. 2002; 249:85-93. 
21. Kobayashi Z, Hino T, Kanazawa T, Yokote H, Yokota T, Kanda T. Bilateral medial medullary infarction presented with monoplegia of the lower limb, followed by paraplegia and finally by tetraplegia. Rinsho Shinkeigaku. 2003;43:195-98.

22. Takano K, Takasugi $\mathrm{k}$. A case of bilateral lower pons-medial medullary infarction presenting quadriparesis. No To Shinkei. 2003;55:879-83. Japanese.

23. Maeda M, Shimono T, Tsukahara H, Maier SE, Takeda K. Acute bilateral medial medullary infarction: a unique 'heart appearance' sign by diffusion-weighted imaging. Eur Neurol. 2004;51:236-7.

24. Jikumaru M, Masuda T, Ueyama H, Sannomiya K, Kumamoto T. Bilateral medial medullary infarction presenting as vertical gaze palsy. Rinsho Shinkeigaku. 2006;6:45-9.

25. Kim JS, Kim HG, Chung CS. Medial medullary syndrome. Report of 18 new patients and a review of the literature. Stroke. 1995;26:1548-52.

26. Takisawa S, Akiyama K, Takagi S, Shinohara Y. Bilateral medial medullary infarction: A case report and review of the literature. Cerebrovasc disease. 1996;6:308-312.

27. Moon SY, Kim HY, Chung CS. A sequential bilateral medial medullary infarction separated by 4 months. Neurology. 2002;59:1814-1815.

28. Alvarez-cermeno JC, Benito-Leon J. Isolated total tongue paralysis as a manifestation of bilateral medullary infarction. J Neurol Neurosurg Psychiatry. 2003;74:1698-9.

29. Gondim Fde A, Cruz-Flores S, Leacock RO. Neuroimaging and pathology of the progression of unilateral to bilateral medial medullary stroke. Cerebrovasc Dis. 2006;22:202-4.

30. Hagiwara N, Toyoda K, Torisu R, Inoue T, Yasumori K, Ibayashi S, et al. Progressive stroke involving bilateral medial medulla expanding to spinal cord due to vertebral artery dissection. Cerebrovasc Dis. 2007;24:540-2.

31. Liu CL, Lin SH, Tetraplegia following bilateral medial medullary infarction. Tzu Chi medical journal. 2009;21:248-250.

32. Krishnan M, Rajan P, Kesavadas C, Iyer RS. The 'heart appearance' sign in MRI in bilateral medial medullary infarction. Postgrad Med J. 2011;87:156-7.

33. ZicklerP,Seitz RJ, Hartung HP, Hefter H. Bilateral medullary pyramid infarction. Neurology. $2005 ; 24 ; 64: 1801$.

34. Tokuoka K, Yuasa N, Ishikawa T, Takahashi M, Mandokoro H, Kitagawa Y, et al. A case of bilateral medial medullary infarction presenting with "heart appearance" sign. Tokai J Exp Clin Med. 2007; 20:99-102.

35. Paliwal VK, Kalita J, Misra UK. Dysphagia in a patient with bilateral medial medullary infarcts. Dysphagia. 2009;24:349-53.

36. Ma L, Deng Y, Wang J, Du F, Xia F, Liu Y, et al. Bilateral medial medullary infarction presenting as Guillain-Barré-like syndrome. Clin Neurol Neurosurg. 2011;113:589-91. 


\section{Legends:}

Figure 1

Figure 1A and 1B: DWI MRI shows restricted diffusion involving bilateral medial medulla ('Heart sign') (arrow) and the right inferior cerebellar hemisphere consistent with an acute infarction.

Figure 1C: MR angiogram demonstrates diffuse intracranial atherosclerosis. The left superior cerebellar artery was occluded on its second segment. There was no significant stenosis or occlusion of the vertebral and basilar arteries. The right PICA, anterior spinal arteries were not visualized. 
Table 1: Clinical characteristics, imaging and prognosis of Bilateral MMI reported in the literature

\begin{tabular}{|c|c|c|c|c|c|c|}
\hline Author & No. & $\begin{array}{c}\text { Age } \\
(\text { yrs }) / \\
\text { sex }\end{array}$ & Year & Clinical presentation & Vascular Imaging & Outcome \\
\hline $\begin{array}{l}\text { Toyoda } \mathrm{K} \\
\text { et al (7) }\end{array}$ & 1 & $71 / \mathrm{M}$ & 1992 & $\begin{array}{l}\text { gaze palsy, } \\
\text { dysarthria, } \\
\text { hypoglossal palsy, } \\
\text { quadriplegia, } \\
\text { horizontal nystagmus } \\
\text { respiratory failure }\end{array}$ & $\begin{array}{l}\text { Left VA and BA } \\
\text { irregularity, } \\
\text { Small aneurysm middle } \\
\text { BA, right VA } \\
\text { hypoplasia and } \\
\text { atherosclerosis, } \\
\text { ASA nonvisualised } \\
\end{array}$ & $\begin{array}{l}\text { persistent } \\
\text { quadriplegia at } \\
2 \text { months } \\
\text { mRS5 }\end{array}$ \\
\hline $\begin{array}{l}\text { Kleinert G } \\
\text { et al (13) }\end{array}$ & 1 & $59 / \mathrm{M}$ & 1993 & $\begin{array}{l}\text { hypoglossal palsy, } \\
\text { quadriplegia, } \\
\text { sensory loss, } \\
\text { respiratory failure }\end{array}$ & $\begin{array}{l}\text { Autopsy showed VA } \\
\text { and BA atherosclerosis } \\
\text { bilaterally with } \\
\text { occlusions in the ASA } \\
\text { branches }\end{array}$ & $\begin{array}{l}\text { Death at } 2 \\
\text { weeks } \\
\text { (pneumonia) }\end{array}$ \\
\hline $\begin{array}{l}\text { Ohashi et } \\
\text { al (14) }\end{array}$ & 1 & $61 / \mathrm{F}$ & 1995 & $\begin{array}{l}\text { hypoglossal palsy } \\
\text { quadriplegia, } \\
\text { sensory loss, }\end{array}$ & NA & NA \\
\hline $\begin{array}{l}\text { Kim JS } \\
(25)\end{array}$ & 1 & 59/M & 1995 & $\begin{array}{l}\text { facial paralysis, } \\
\text { dysarthria, } \\
\text { dysphagia } \\
\text { motor deficit, } \\
\text { paresthesia, } \\
\text { impair vibration } \\
\text { upbeat nystagmus } \\
\end{array}$ & VA occlusion & NA \\
\hline $\begin{array}{l}\text { Roh JK et } \\
\text { al (15) }\end{array}$ & 1 & $71 / \mathrm{M}$ & 1996 & $\begin{array}{l}\text { impair deep sensation } \\
\text { Sensory ataxia, } \\
\text { upbeat nystagmus } \\
\text { bilateral dysmetria, } \\
\text { trucal ataxia }\end{array}$ & VA and BA normal & $\begin{array}{l}\text { Stand and walk } \\
\text { with } 2 \text { weeks } \\
\text { mRS } 3\end{array}$ \\
\hline $\begin{array}{l}\text { Wada K et } \\
\text { al (8) }\end{array}$ & 1 & $69 / \mathrm{M}$ & 1996 & $\begin{array}{l}\text { gaze palsy, } \\
\text { bilateral facial } \\
\text { weakness, } \\
\text { left hemiparesis, } \\
\text { horizontal nystagmus } \\
\end{array}$ & Right VA occlusion & NA \\
\hline $\begin{array}{l}\text { Toyoda } \mathrm{K} \\
\text { et al (3) }\end{array}$ & 1 & 71/M & 1996 & $\begin{array}{l}\text { bilateral facial palsy, } \\
\text { dysarthria, } \\
\text { right hypoglossal palsy, } \\
\text { right hemiparesis, } \\
\text { bilateral sensory loss, } \\
\text { upward and horizontal } \\
\text { nystagmus }\end{array}$ & $\begin{array}{l}\text { Right VA hypoplasia, } \\
\text { Left VA } \\
\text { athrosclerosis(wall } \\
\text { irregularity) }\end{array}$ & $\begin{array}{l}\text { mRS } 5 \text { at } 2 \\
\text { months }\end{array}$ \\
\hline $\begin{array}{l}\text { Toyoda K } \\
\text { et al (3) }\end{array}$ & 1 & $47 / \mathrm{M}$ & 1996 & $\begin{array}{l}\text { quadriparesis, } \\
\text { bilateral sensory loss, } \\
\text { horizontal nystagmus }\end{array}$ & $\begin{array}{l}\text { Normal angiogram with } \\
\text { nonvisualised ASA }\end{array}$ & $\begin{array}{l}\text { mRS } 3 \text { at } 2 \\
\text { months }\end{array}$ \\
\hline $\begin{array}{l}\text { Takizawa } \\
\text { S et al(26) }\end{array}$ & 1 & $70 / \mathrm{M}$ & 1996 & $\begin{array}{l}\text { dysarthria, } \\
\text { dysphagia, } \\
\text { bilateral hypoglossal } \\
\text { palsy, quadriplegia } \\
\text { gazed evoke nystagmus }\end{array}$ & $\begin{array}{l}\text { Normal VA and BA, } \\
\text { Nonvisualised ASA }\end{array}$ & $\begin{array}{l}\text { mRS4 at } 7 \\
\text { months }\end{array}$ \\
\hline
\end{tabular}




\begin{tabular}{|c|c|c|c|c|c|c|}
\hline $\begin{array}{l}\text { Del Brutto } \\
\text { OH et } \\
\mathrm{al}(16)\end{array}$ & 1 & $58 / \mathrm{M}$ & 1998 & $\begin{array}{l}\text { hypoglossal palsy } \\
\text { quadriplegia, }\end{array}$ & $\begin{array}{l}\text { VA partial occlude, } \\
\text { VA dolichoectasia }\end{array}$ & $\begin{array}{l}\text { Quadr iplegia } \\
\text { at } 2 \text { months } \\
\text { mRS5 }\end{array}$ \\
\hline $\begin{array}{l}\text { Bailbe M } \\
\text { et al (17) }\end{array}$ & 1 & $42 / \mathrm{M}$ & 2000 & NA & NA & $\begin{array}{l}\text { Favorable } \\
\text { outcome }\end{array}$ \\
\hline $\begin{array}{l}\text { Katoh M } \\
\text { et al (18) }\end{array}$ & 1 & $71 / \mathrm{M}$ & 2000 & $\begin{array}{l}\text { horizontal gaze palsy, } \\
\text { dysarthria, } \\
\text { impaired gag reflex, } \\
\text { quadriparesis, }\end{array}$ & NA & $\begin{array}{l}5 \text { mth able to } \\
\text { walk,mRS } 3 \\
\text { Hemiparesis at } \\
1 \text { year, }\end{array}$ \\
\hline $\begin{array}{l}\text { Thijs RD } \\
\text { et al (19) }\end{array}$ & 1 & $56 / \mathrm{M}$ & 2001 & $\begin{array}{l}\text { dysarthria, } \\
\text { quadriplegia, } \\
\text { respiratory failure }\end{array}$ & $\begin{array}{l}\text { Right VA occlusion, } \\
\text { Non visualized ASA }\end{array}$ & $\begin{array}{l}\text { Quadriplegia at } \\
2 \text { months } \\
\text { mRS } 5\end{array}$ \\
\hline $\begin{array}{l}\text { Kumral E } \\
\text { et al (20) }\end{array}$ & 1 & $57 / \mathrm{M}$ & 2002 & $\begin{array}{l}\text { facial palsy,dysarthria } \\
\text { hypoglossal palsy } \\
\text { quadriplegia, } \\
\text { sensory loss, } \\
\text { upbeat, horizontal, } \\
\text { rotatory nystagmus, } \\
\text { gait ataxia }\end{array}$ & VA dolichoectasia & $\begin{array}{l}\text { Quadriplegia at } \\
3 \text { months } \\
\text { mRS5 }\end{array}$ \\
\hline $\begin{array}{l}\text { Moon SY } \\
\text { et al (27) }\end{array}$ & 1 & $81 / \mathrm{F}$ & 2002 & $\begin{array}{l}\text { anarthria,hypoglossal } \\
\text { palsy,quidriplegia, } \\
\text { impair vibration }\end{array}$ & $\begin{array}{l}\text { Normal VA and BA, } \\
\text { ASA non visualized }\end{array}$ & $\begin{array}{l}\text { Death, } \\
\text { (aspiration } \\
\text { pneumonia) }\end{array}$ \\
\hline $\begin{array}{l}\text { Kobayashi } \\
Z \text { et al } \\
(21)\end{array}$ & 1 & $69 / \mathrm{M}$ & 2003 & quadriplegia & NA & NA \\
\hline $\begin{array}{l}\text { Takano K } \\
\text { et al (22) }\end{array}$ & 1 & $63 / \mathrm{F}$ & 2003 & $\begin{array}{l}\text { dysarthria, } \\
\text { quadriparesis, } \\
\text { sensory loss, } \\
\text { horizontal nystagmus } \\
\end{array}$ & $\begin{array}{l}\text { Normal angiogram, } \\
\text { ASA nonvisualised }\end{array}$ & $\begin{array}{l}\text { Quadriplegia at } \\
2 \text { years, mRS5 }\end{array}$ \\
\hline $\begin{array}{l}\text { Alvarez- } \\
\text { Cermeno } \\
\text { Jc et al } \\
(28)\end{array}$ & 1 & $49 / \mathrm{F}$ & 2003 & $\begin{array}{l}\text { Bilateral hypoglossal } \\
\text { palsy }\end{array}$ & $\begin{array}{l}\text { VA atherosclerosis, } \\
\text { BA stenosis }\end{array}$ & $\begin{array}{l}\text { Favorable } \\
\text { outcome at } 2 \\
\text { months, mRS } 1\end{array}$ \\
\hline $\begin{array}{l}\text { Kameda } \\
\text { W et al } \\
(12)\end{array}$ & 6 & NA & 2004 & $\begin{array}{l}\text { sensory loss, horner's } \\
\text { syndrome, cerebellar } \\
\text { ataxia }\end{array}$ & $\begin{array}{l}\text { VA and PICA } \\
\text { atherosclerosis } \\
\text { (number patient }=2 \text { ) }\end{array}$ & $\mathrm{NA}$ \\
\hline $\begin{array}{l}\text { Fukuda M } \\
\text { et al (9) }\end{array}$ & 1 & $52 / \mathrm{F}$ & 2004 & $\begin{array}{l}\text { hypoglossal palsy, } \\
\text { hemiplegia, } \\
\text { sensory loss }\end{array}$ & $\begin{array}{l}\text { VA dissection } \\
\text { bilaterally }\end{array}$ & $\begin{array}{l}\text { Hemiplegia at } \\
18 \text { months, } \\
\text { mRS3 }\end{array}$ \\
\hline $\begin{array}{l}\text { Maeda M } \\
\text { et al (23) }\end{array}$ & 1 & $69 / \mathrm{F}$ & 2004 & $\begin{array}{l}\text { dysarthria, } \\
\text { quadriplegia, } \\
\text { horizontal nystagmus, } \\
\text { respiratory failure }\end{array}$ & VA stenosis/ occlusion & NA \\
\hline $\begin{array}{l}\text { Zickler P } \\
\text { et al (33) }\end{array}$ & 1 & NA & 2005 & $\begin{array}{l}\text { Quadriparesis, } \\
\text { autonomic dysregulation }\end{array}$ & NA & NA \\
\hline $\begin{array}{l}\text { Jikumaru } \\
\text { et al (24) }\end{array}$ & 1 & $79 / \mathrm{M}$ & 2006 & $\begin{array}{l}\text { gaze palsy, } \\
\text { Left facial palsy, } \\
\text { dysarthria, } \\
\text { quadriplegia, respiratory } \\
\text { failure }\end{array}$ & NA & NA \\
\hline
\end{tabular}




\begin{tabular}{|c|c|c|c|c|c|c|}
\hline $\begin{array}{l}\text { Gondim F } \\
\text { et al (29) }\end{array}$ & 1 & $64 / \mathrm{F}$ & 2006 & $\begin{array}{l}\text { hypoglossal palsy, } \\
\text { quardriparesis, } \\
\text { upbeat nystagmus, } \\
\text { respiratory failure }\end{array}$ & $\begin{array}{l}\text { Right VA stenosis, } \\
\text { BA stenosis( } 50 \%)\end{array}$ & $\begin{array}{l}\text { Death at } 2 \\
\text { months } \\
\text { (r/o peritonitis) }\end{array}$ \\
\hline $\begin{array}{l}\text { Tokuoka } \\
\text { K et al } \\
(34)\end{array}$ & 1 & $62 / \mathrm{M}$ & 2007 & $\begin{array}{l}\text { dysarthria, dysphagia, } \\
\text { Quardriplegia, } \\
\text { Sensory loss }\end{array}$ & NA & mRS 5 \\
\hline $\begin{array}{l}\text { Paliwal } \\
\text { VK et al } \\
\text { (35) }\end{array}$ & 1 & $70 / \mathrm{M}$ & 2009 & $\begin{array}{l}\text { anarthria, dysphagia, } \\
\text { quardriplegia, } \\
\text { gaze evoke nystagmus, } \\
\text { respiratory failure }\end{array}$ & $\begin{array}{l}\text { Hypoplastic right VA, } \\
\text { ASA non visualised }\end{array}$ & $\begin{array}{l}\text { Death, } \\
\text { (pneumonia) }\end{array}$ \\
\hline $\begin{array}{l}\text { Kim JS et } \\
\text { al (6) }\end{array}$ & 12 & NA & 2009 & $\begin{array}{l}\text { dysarthria, } \\
\text { quadriparesis, } \\
\text { nystagmus }\end{array}$ & $\begin{array}{l}\text { atheromatous branch } \\
\text { occlusion }\end{array}$ & NA \\
\hline $\begin{array}{l}\text { Liu CL et } \\
\text { al (31) }\end{array}$ & 1 & $54 / \mathrm{F}$ & 2009 & $\begin{array}{l}\text { dysphagia, hiccough, } \\
\text { quardriparesis, } \\
\text { impair vibration }\end{array}$ & Normal VA and BA & $\begin{array}{l}\text { Mechanical } \\
\text { ventilation/ } \\
\text { persistent } \\
\text { quadriplegia at } \\
1 \text { year, mRS5 } \\
\end{array}$ \\
\hline $\begin{array}{l}\text { Shono Y } \\
\text { et al (5) }\end{array}$ & 1 & $57 / \mathrm{M}$ & 2010 & $\begin{array}{l}\text { facial palsy, } \\
\text { hypoglossal palsy, } \\
\text { hemiparesis }\end{array}$ & $\begin{array}{l}\text { Normal VA and BA, } \\
\text { ASA nonvisualised }\end{array}$ & $\begin{array}{l}\text { mRS } 2 \text { at } 3 \\
\text { months }\end{array}$ \\
\hline $\begin{array}{l}\text { Shono Y } \\
\text { et al (5) }\end{array}$ & 1 & $60 / \mathrm{M}$ & 2010 & $\begin{array}{l}\text { dysarthria, } \\
\text { bilateral hypoglossal, } \\
\text { palsy, quadriparesis, } \\
\text { bilateral sensory } \\
\text { dysfunction, ataxia }\end{array}$ & $\begin{array}{l}\text { Left VA occlusion after } \\
\text { Branching } \\
\text { PICA(dissection) }\end{array}$ & $\begin{array}{l}\text { mRS } 5 \text { at } 3 \\
\text { months }\end{array}$ \\
\hline $\begin{array}{l}\text { Krishnan } \\
\text { M et } \\
\text { al(32) }\end{array}$ & 1 & $70 / \mathrm{M}$ & 2011 & $\begin{array}{l}\text { dysarthria, } \\
\text { hypoglossal palsy, } \\
\text { quadriparesis, } \\
\text { impair vibratory, } \\
\text { horizontal nystagmus, } \\
\text { respiratory failure }\end{array}$ & $\begin{array}{l}\text { Bilateral VA and BA } \\
\text { atherosclerosis }\end{array}$ & $\begin{array}{l}\text { Residual } \\
\text { spastic } \\
\text { quadriparesis } \\
\text { at } 1 \text { month, } \\
\text { mRS5 }\end{array}$ \\
\hline $\begin{array}{l}\text { Ma L et al } \\
\text { (36) }\end{array}$ & 1 & $49 / \mathrm{M}$ & 2011 & $\begin{array}{l}\text { dysarthria,quadriplegia, } \\
\text { horizontal nystagmus }\end{array}$ & Normal VA, BA & $\begin{array}{l}\text { Motor power } \\
\text { gr } 3 / 5 \text { at } 18 \\
\text { months,mRS5 }\end{array}$ \\
\hline $\begin{array}{l}\text { Ma L et al } \\
\text { (36) }\end{array}$ & 1 & $48 / \mathrm{M}$ & 2011 & $\begin{array}{l}\text { dysarthria, dysphagia } \\
\text { hypoglossal palsy, } \\
\text { Quadriparesis, } \\
\text { bilateral sensory loss } \\
\text { horizontal nystagmus, }\end{array}$ & Left VA occlusion & $\begin{array}{l}\text { Motor power } \\
\text { gr } 4 / 5 \text { at } 1 \mathrm{mth} . \\
\text { mRS } 1 \text { at } 15 \\
\text { months }\end{array}$ \\
\hline $\begin{array}{l}\text { Present } \\
\text { case }\end{array}$ & 1 & $71 / \mathrm{M}$ & 2010 & $\begin{array}{l}\text { gaze palsy, dysarthria, } \\
\text { quadriplegia, } \\
\text { Respiratory failure, }\end{array}$ & $\begin{array}{l}\text { Normal VA and BA } \\
\text { ASA nonvisualized }\end{array}$ & $\begin{array}{l}\text { Death } 1 \text { month } \\
\text { (pneumonia) }\end{array}$ \\
\hline
\end{tabular}

Abbreviations: M- male; F-female; VA- vertebral artery; BA- basilar artery; ASA- anterior spinal artery; PICA- posterior inferior cerebellar artery; mRS- modified Rankin scale; NA- details not available 
Table 2. Clinical Manifestations

\begin{tabular}{|c|c|c|}
\hline Symptoms and signs (Total n=37) & Number & $(\%)$ \\
\hline Motor & 29 & $(78.4)$ \\
\hline Bilateral weakness & 24 & $(64.9)$ \\
\hline Hemiparesis & 4 & $(10.8)$ \\
\hline Unidentified & 1 & $(2.7)$ \\
\hline Dysarthria & 18 & $(48.6)$ \\
\hline Nystagmus & 18 & (48.6) \\
\hline Upbeat & 5 & (13.5) \\
\hline Horizontal & 10 & (27) \\
\hline Gaze evoked & 2 & (5.4) \\
\hline Rotatory & 1 & (2.7) \\
\hline Sensory & 16 & $(43.2)$ \\
\hline Hypoglossal palsy & 15 & $(40.5)$ \\
\hline Bilateral & 3 & $(8.1)$ \\
\hline Unilateral & 1 & $(2.7)$ \\
\hline Unidentified & 11 & (29.7) \\
\hline Respiratory failure & 9 & (24.3) \\
\hline Dysphagia & 6 & (16.2) \\
\hline Facial palsy & 6 & $(16.2)$ \\
\hline Gaze palsy & 5 & (13.5) \\
\hline Ataxia & 4 & (10.8) \\
\hline Horner Syndrome & 1 & (2.7) \\
\hline
\end{tabular}




\section{Table 3. Vascular Imaging}

Vascular Pathology (Totol n=26)

Normal VA, BA, ASA

VA atherosclerosis

VA occlusion

BA atherosclerosis

Dissection

VA dolichoectasia

ASA occlusion

PICA atherosclerosis $\mathrm{n}$

$(\%)$

10

4

5

(19.2)

2

2

1

2 Abbreviations: VA- vertebral artery; BA- basilar artery; ASA- anterior spinal artery; PICA- posterior inferior cerebellar artery
From the case report some patients have more than one vascular pathology.

Note: Total percentage is higher than 100 due to more than one vessel affected in some cases. 
Figure 1- Illustrative case

A)

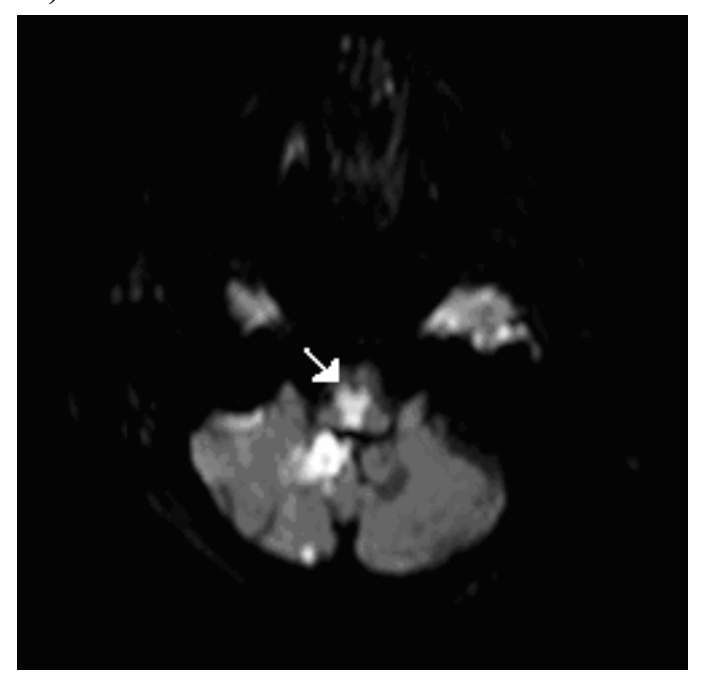

C)

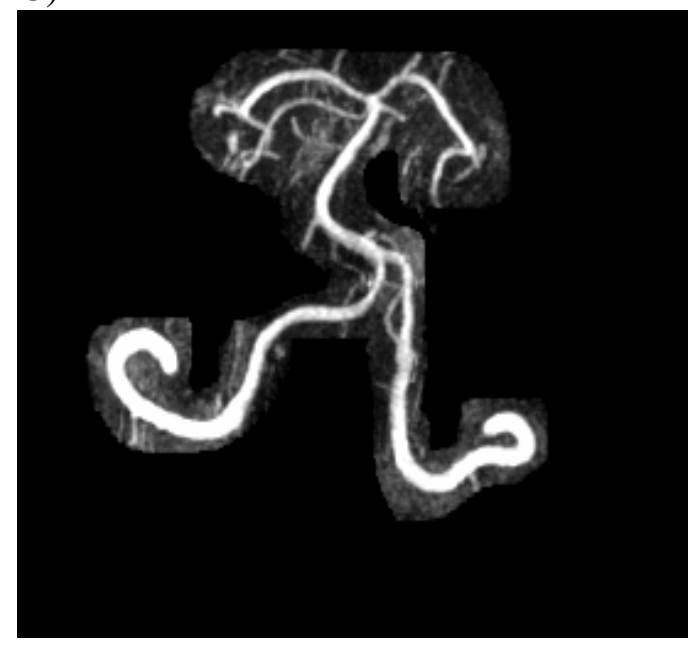

B)

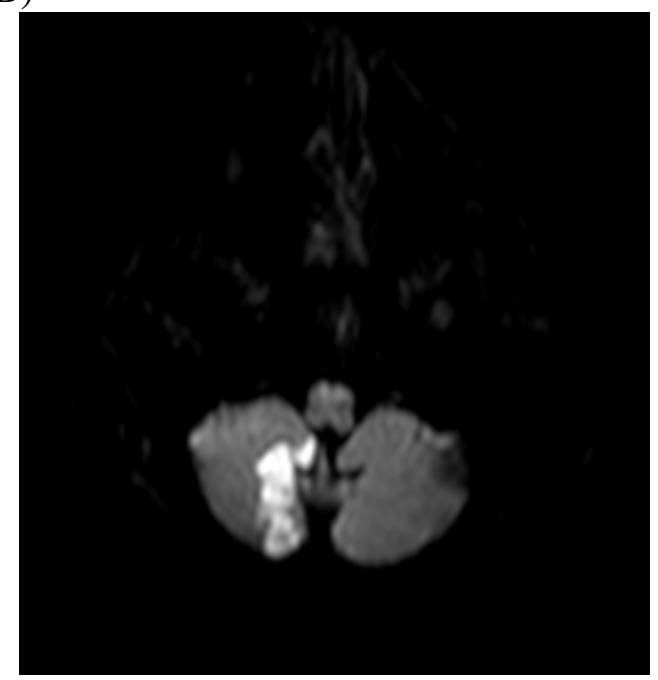

Figure 1A and 1B: DWI MRI shows restricted diffusion involving bilateral medial medulla ('Heart sign') (arrow) and the right inferior cerebellar hemisphere consistent with an acute infarction.

Figure 1C: MR angiogram demonstrates diffuse intracranial atherosclerosis. The left superior cerebellar artery was occluded on its second segment. There was no significant stenosis or occlusion of the vertebral and basilar arteries. The right PICA, anterior spinal arteries were not visualized. 